\title{
Cones based on reflection symmetric convex polygons: Remarks on a problem by A. Pleijel
}

\author{
Marino Belloni * $\quad$ Dirk Horstmann ${ }^{\dagger} \quad$ Bernd Kawohl ${ }^{\ddagger}$
}

March 16, 2006

\begin{abstract}
In this note we investigate a problem formulated by Pleijel in 1955. It asks for the cone over a convex plane domain $\mathcal{K}$ having minimal surface among all cones over $\mathcal{K}$ with the same given height $h$. For cones based on reflection symmetric polygonal $\mathcal{K}$ we analyze the behaviour, as $h \rightarrow 0$ and as $h \rightarrow \infty$, of the position of the apex for the minimizing cone and characterize the coordinate of the limit points by necessary conditions. Furthermore, the question whether there are convex domains such that the minimal cone does not change with $h$ is discussed. The results about the location of the optimal point in the limits $h \rightarrow \infty$ and $h \rightarrow 0$ presented here give the more or less explicit algebraic coordinates of the optimal apex. A complete (but implicit) characterization of this point was given by B. Cheng in [1].
\end{abstract}

Keywords: Convex domains, optimization problem, minimal surfaces

2000 MSC numbers: 51M04, 51M15, 51M25, 52A10, 52A38, 52B15, 53A05

\section{Introduction and formulation of the problem}

In the book "Unsolved problems in geometry" by Croft, Falconer and Guy [2, page 41] one can find the following problem, which was originally stated by A. Pleijel [3] in 1955.

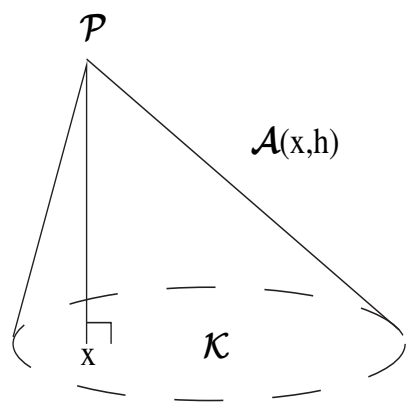

Figure 1: For which $x^{*}(h)$ is $\mathcal{A}(x(h), h)$ minimal?

*Dipartimento di Matematica, Università di Parma, 43100 Parma, Italy, email: marino.belloni@unipr.it

${ }^{\dagger}$ Mathematisches Institut der Universität zu Köln, D-50923 Köln, Germany, email: dhorst@math.uni-koeln.de

${ }^{\ddagger}$ Mathematisches Institut der Universität zu Köln, D-50923 Köln, Germany, email: kawohl@math.uni-koeln.de 
"Let $\mathcal{K}$ be a convex set contained in a plane $P$ in 3-dimensional space. Given $h>0$ and a point $x(h)$ in $\mathcal{K}$, from the cone based on $\mathcal{K}$ with vertex distance $h$ up the perpendicular to $P$ through $x(h)$, and let $\mathcal{A}(x(h), h)$ denote the surface area of this cone. For fixed $h$ there is a unique $x^{*}(h)$ for which $\mathcal{A}(x(h), h)$ attains a minimum. How are the points $x^{*}(h)$ related to $\mathcal{K}$ ? If $\mathcal{K}$ is any polygon circumscribing a circle, then $x^{*}(h)$ is the center of the circle. Is there any simple description of these points for other plane convex sets? In particular, is there a simple description of $\lim _{h \rightarrow 0} x^{*}(h)$ and $\lim _{h \rightarrow \infty} x^{*}(h)$ in terms of the geometry of $\mathcal{K}$ ?"

Remark 1 A polygon is circumscribing a circle if and only if each tangent line to a side of the polygon is tangential to the circle. However, the sides do not have to touch the circumscribed circle (see for example Figure 3).

The present paper will give some answers to this question by A. Pleijel for reflection symmetric polygons. Independent of the shape of the plane domain $\mathcal{K}$ one might consider this question as the minimizing problem

$$
\min _{x(h) \in \mathcal{K}} \mathcal{A}(x(h), h)
$$

for a fixed height $h$. This is in fact a minimizing problem over a convex domain in $\mathbb{R}^{2}$ and - at first glance - it seems to be solvable without great difficulties. But is there an explicit easy-to-handle formula for $\mathcal{A}(x(h), h)$ ? In other words, is there a possibility to express the cone as the graph of a function $u(x(h), h, \xi)$, with $\xi=\left(\xi_{1}, \xi_{2}\right) \in \mathcal{K}$, for given height $h$ and a fixed point $x(h)$ ? Knowing $u(x(h), h, \xi)$, the surface of the cone is given by

$$
\int_{\mathcal{K}} \sqrt{1+\left|D_{\xi} u\right|^{2}} d \xi
$$

which is for a convex set $\mathcal{K}$ a strict convex function in $x(h) \in \mathbb{R}^{2}$. This can be seen as follows.
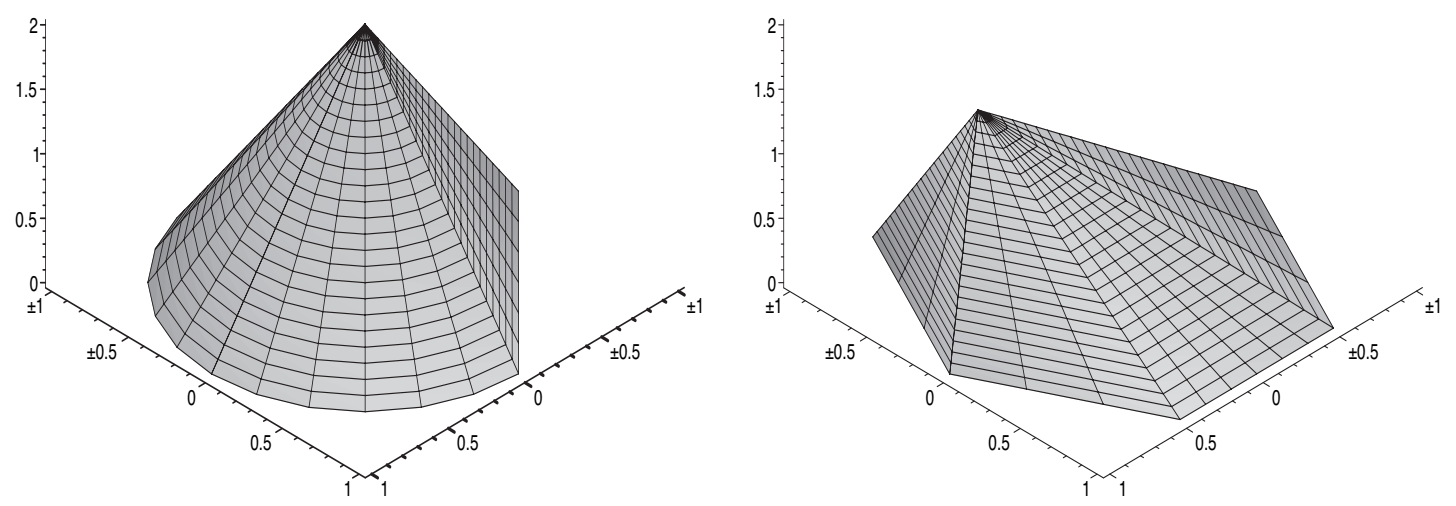

Figure 2: Cones over convex sets.

If the boundary $\partial \mathcal{K}$ of the convex domain is smooth enough, let us say of class $C^{1}$, then the cone can be described by using a parametrization of $\partial \mathcal{K}$. We describe the cone of vertex $P_{h}=\left(x_{1}(h), x_{2}(h), h\right)$ lying on a parametric curve $\Gamma(t), t \in[0,2 \pi]$ (the boundary of $\mathcal{K}$ ), with the help of its arc length parametrization $\gamma(s)=\left(\gamma_{1}(s), \gamma_{2}(s), 0\right), s \in[0, l]\left(d s=\left|\Gamma^{\prime}(t)\right| d t\right)$, where $l$ is the length of $\Gamma(t)$. In parametric form this cone is then given by

$$
f(s, q)=q P_{h}+(1-q) \gamma(s)=\left(\begin{array}{c}
q x_{1}(h)+(1-q) \gamma_{1}(s) \\
q x_{2}(h)+(1-q) \gamma_{2}(s) \\
q h
\end{array}\right)
$$


for $s \in[0, l], q \in[0,1]$. We compute the differential

$$
D f(s, q)=\left(\begin{array}{cc}
(1-q) \gamma_{1}^{\prime}(s) & x_{1}(h)-\gamma_{1}(s) \\
(1-q) \gamma_{2}^{\prime}(s) & x_{2}(h)-\gamma_{2}(s) \\
0 & h
\end{array}\right)
$$

and the Jacobian $J(f)$, given by

$$
J^{2}(f)=\left[(1-q) \gamma_{1}^{\prime}(s)\left(x_{2}(h)-\gamma_{2}(s)\right)-(1-q) \gamma_{2}^{\prime}(s)\left(x_{1}(h)-\gamma_{1}(s)\right)\right]^{2}+\left[h(1-q) \gamma_{1}^{\prime}(s)\right]^{2}+\left[h(1-q) \gamma_{2}^{\prime}(s)\right]^{2} .
$$

Then the area formula becomes

$$
\mathcal{A}(x(h), h)=\int_{0}^{1} d q \int_{0}^{l}(1-q) \sqrt{h^{2}+\left|\left[\left(x_{1}(h), x_{2}(h)\right)-\left(\gamma_{1}(s), \gamma_{2}(s)\right)\right] n(s)\right|^{2}} d s,
$$

where $n(s):=\left(-\gamma_{2}^{\prime}(s), \gamma_{1}^{\prime}(s)\right)$ with $\gamma_{1}^{\prime 2}(s)+\gamma_{2}^{\prime 2}(s)=1$. Evaluating the integral with respect to $q$, we have

$$
\mathcal{A}(x(h), h)=\frac{1}{2} \int_{0}^{l} \sqrt{h^{2}+\left|\left[\left(x_{1}(h), x_{2}(h)\right)-\left(\gamma_{1}(s), \gamma_{2}(s)\right)\right] n(s)\right|^{2}} d s .
$$

However, it is not clear whether it is possible to write the previous expression in terms of elementary functions. But from this formula it is possible to observe for a convex set, that this functional is strictly convex in $x(h)=\left(x_{1}(h), x_{2}(h)\right)$, as it can be seen by the following calculations. Set

$$
g\left(x_{1}, x_{2}, s\right):=\sqrt{h^{2}+\left|\left[\left(x_{1}, x_{2}\right)-\left(\gamma_{1}(s), \gamma_{2}(s)\right)\right] n(s)\right|^{2}} .
$$

Then

$$
g_{i, j}\left(x_{1}, x_{2}, s\right):=\frac{\partial^{2} g}{\partial x_{j} \partial x_{j}}=\frac{h}{\left(h^{2}+\left|\left[\left(x_{1}, x_{2}\right)-\left(\gamma_{1}(s), \gamma_{2}(s)\right)\right] n(s)\right|^{2}\right)^{3 / 2}} \gamma_{i}^{\prime}(s) \gamma_{j}^{\prime}(s)
$$

Now

$$
\mathcal{A}\left(x_{1}, x_{2}, h\right)=\frac{1}{2} \int_{0}^{l} g\left(x_{1}, x_{2}, h\right) d s \text { and its Hessian is given by } H_{\mathcal{A}}\left(x_{1}, x_{2}\right)=\left(\frac{1}{2} \int_{0}^{l} g_{i, j}\left(x_{1}, x_{2}, s\right) d s\right)_{i, j=1}^{2} .
$$

We observe that

$$
\operatorname{trace} H_{\mathcal{A}}\left(x_{1}, x_{2}\right)=\frac{1}{2} \int_{0}^{l}\left(g_{1,1}\left(x_{1}, x_{2}, s\right)+g_{2,2}\left(x_{1}, x_{2}, s\right)\right) d s>0
$$

and

$$
\operatorname{det} H_{\mathcal{A}}\left(x_{1}, x_{2}\right)=\left(\frac{1}{2} \int_{0}^{l} g_{1,1}\left(x_{1}, x_{2}, s\right) d s\right)\left(\frac{1}{2} \int_{0}^{l} g_{2,2}\left(x_{1}, x_{2}, s\right) d s\right)-\left(\frac{1}{2} \int_{0}^{l} g_{1,2}\left(x_{1}, x_{2}, s\right) d s\right)^{2}>0 .
$$

The last inequality holds true because of the Hölder estimate

$$
\begin{aligned}
\left(\frac{1}{2} \int_{0}^{l} g_{1,2}\left(x_{1}, x_{2}, s\right) d s\right)^{2} \leq & \left(\frac{1}{2} \int_{0}^{l} \frac{h}{\left(h^{2}+\left|\left[\left(x_{1}, x_{2}\right)-\left(\gamma_{1}(s), \gamma_{2}(s)\right)\right] n(s)\right|^{2}\right)^{3 / 2}} \gamma_{1}^{\prime 2}(s) d s\right) \times \\
& \times\left(\frac{1}{2} \int_{0}^{l} \frac{h}{\left(h^{2}+\left|\left[\left(x_{1}, x_{2}\right)-\left(\gamma_{1}(s), \gamma_{2}(s)\right)\right] n(s)\right|^{2}\right)^{3 / 2}} \gamma_{2}^{\prime 2}(s) d s\right)
\end{aligned}
$$


and the equality holds if and only if there exists $\lambda \neq 0$ such that, for every $s \in[0, l]$,

$$
\frac{h^{1 / 2} \gamma_{1}^{\prime}(s)}{\left(h^{2}+\left|\left[\left(x_{1}, x_{2}\right)-\left(\gamma_{1}(s), \gamma_{2}(s)\right)\right] n(s)\right|^{2}\right)^{3 / 4}}=\lambda \frac{h^{1 / 2} \gamma_{2}^{\prime}(s)}{\left(h^{2}+\left|\left[\left(x_{1}, x_{2}\right)-\left(\gamma_{1}(s), \gamma_{2}(s)\right)\right] n(s)\right|^{2}\right)^{3 / 4}}
$$

For a convex set $\mathcal{K}$ the last condition cannot hold (unless $\mathcal{K}$ is a line segment). Therefore, both eigenvalues are strictly positive, and the function is strictly convex. Obviously, when $h \rightarrow 0$ both eigenvalues disappear.

Since $\mathcal{A}(x(h), h)$ is strictly convex on bounded convex domains, the uniqueness of a minimal point $x^{*}(h)$ is guaranteed. Furthermore, for reflection symmetric convex domains with only one axis of symmetry the strict convexity implies that the optimal point $x^{*}(h)$ must lie on the symmetry axis for every fixed $h$. Thus, $x^{*}(h)$ is either in the interior of $\mathcal{K}$ or coincides with one of the intersection points of the symmetry axis with the boundary of $\mathcal{K}$.

In his paper "On a problem of A. Pleijel" B. N. Cheng [1] locates the limiting point as $h \rightarrow 0$ and as $h \rightarrow \infty$ implicitly via a special boundary parametrization and the radius-of-curvature function $\rho$ of $\partial \mathcal{K}$. Using variational arguments he shows that the unique point $x^{*}(h)$ approaches the origin of the polar coordinate parametrization of $\partial \mathcal{K}$ whose support function $\lambda(\phi)$ is characterized by

$$
\int_{0}^{2 \pi} \frac{\rho}{\lambda^{2}(\phi)} e^{i \phi} d \phi=0 \text { as } h \rightarrow 0 \text { and by } \int_{0}^{2 \pi} \rho \lambda(\phi) e^{i \phi} d \phi=0 \text { as } h \rightarrow \infty .
$$

Furthermore, he shows that these characterizations are sufficient to determine the limiting points, if the domain is strictly convex. The disadvantage of this result lies in the fact that it does not explicitly give the coordinates of the optimal points.

However, it is possible to use Cheng's result to charaterize those smooth convex domains, for which the optimal point has to be independent of the height $h$. For those domains we conclude from Cheng's characterizations that

$$
\int_{0}^{2 \pi} \frac{\rho}{\lambda^{2}(\phi)} e^{i \phi} d \phi=\int_{0}^{2 \pi} \rho \lambda(\phi) e^{i \phi} d \phi
$$

has to hold. Now we express the radius of curvature $\rho=1 / \kappa$ in terms of the support function $\lambda(\phi)$ as $\rho=\lambda(\phi)+\lambda^{\prime \prime}(\phi)$ (where $\lambda(\phi)+\lambda^{\prime \prime}(\phi)>0$ for a convex set). Therefore, in this case,

$$
\int_{0}^{2 \pi} \frac{\lambda(\phi)+\lambda^{\prime \prime}(\phi)}{\lambda^{2}(\phi)} e^{i \phi} d \phi=\int_{0}^{2 \pi}\left(\lambda(\phi)+\lambda^{\prime \prime}(\phi)\right) \lambda(\phi) e^{i \phi} d \phi
$$

has to hold, which is true e.g. if $\lambda^{3}(\phi)=1$, i.e. if $\mathcal{K}$ is a disc. To be precise, for smooth convex domains the optimal point is independent of the given height $h$, if the support function $\lambda(\phi)$ of $\mathcal{K}$ solves the differential equation:

$$
\left(\lambda(\phi)+\lambda^{\prime \prime}(\phi)\right)\left(\frac{1}{\lambda^{2}(\phi)}-\lambda(\phi)\right)=F(\phi)
$$

where $F(\phi)$ has a Fourier expansion of the form

$$
F(\phi)=\lambda_{0}+\sum_{k \geq 2} c_{k} \cos (k \phi)+d_{k} \sin (k \phi) .
$$

Note that the Fourier expansion has no term for $k=1$. Aside from the result of B. Cheng it seems rather impossible to get more information from this variational approach. 


\section{Some useful definitions and first observations}

The goal of the present paper is to localize the optimal points for some special domains explicitly. For the sake of clarity, we start with some special cases and extend these results in the forthcoming sections. Let us introduce the following definitions first.

Definition 1 Any circle of maximal radius that is completely contained in $\mathcal{K}$ is called incircle.

Definition 2 The CMIC-set (centers of maximal inscribed circles) is defined as the set of all points $\hat{x}$ in $\mathcal{K}$ satisfying $\operatorname{dist}(\hat{x}, \partial \mathcal{K})=\max _{x \in \mathcal{K}} \operatorname{dist}(x, \partial \mathcal{K})$.

Remark 2 For a convex domain $\mathcal{K}$ the CMIC-set is convex. Furthermore, the existence of a CMIC-set that is not a singleton implies that there is a straight line segment $\Gamma_{1}$ of $\partial \mathcal{K}$, which is reflected along the CMIC-set to a second part $\Gamma_{2}$ of $\partial \mathcal{K}$ parallel to $\Gamma_{1}$.

Direct consequences from these definitions, the convexity of the domain and the uniqueness of the optimal point for each fixed height $0<h<\infty$, are the following:

1. Suppose the domain $\mathcal{K}$ is centrally symmetric, then $x^{*}(h)$ coincides with the center of symmetry of the domain and therefore it is independent of the height $h$. (See also Remark C in [1, p. 142].)

2. Suppose the domain $\mathcal{K}$ has only one axis of symmetry, then $x^{*}(h)$ lies on this axis but might depend on the height $h$. Furthermore the CMIC-set lies on this axis, too.

These observations give the explicit location of the optimal point and thus characterize the minimizing cone for a large class of convex domains, containing circles, ellipses, rectangles and for example the so-called stadium.

\section{Reflection symmetric convex polygons}

Let us now denote the coordinate unit frame in $\mathbb{R}^{3}$ with $\vec{e}_{1}, \vec{e}_{2}, \vec{e}_{3}$. If $\mathcal{K}$ is a spatial closed polygon the surface area of a cone over $\mathcal{K}$ with apex in $X:=\left(x_{1}, x_{2}, h\right)=(x(h), h) \in \mathbb{R}^{3}$ is given by the formula

$$
\mathcal{A}(x(h), h):=\mathcal{A}(X)=\frac{1}{2} \sum_{i=1}^{k}\left\|\left(\vec{y}_{i}-\vec{x}\right) \otimes \vec{d}_{i}\right\|,
$$

where $y_{i}$ denote the vertices of $\mathcal{K}$ for $i \in\{1, \ldots, k\}, \vec{y}_{i}$ is the position vector of $y_{i}, \vec{d}_{i}:=\vec{y}_{i+1}-\vec{y}_{i}$ is the direction vector of the edge $\overline{y_{i} y_{i+1}}$, and $\vec{x}$ is the position vector of the apex $X . \vec{a} \otimes \vec{b}$ denotes the vector product of the two vectors $\vec{a}$ and $\vec{b}$. With $\|\cdot\|$ we denote the Euclidean norm of a vector in $\mathbb{R}^{3}$. The closedness of the boundary $\partial \mathcal{K}$ implies

$$
\sum_{i=1}^{k} \vec{d}_{i}=0
$$

To see what impact a variation of $X$ has on the surface of the cone we concentrate on one triangle first. The surface of a triangle is

$$
f_{X}=\frac{1}{2}\left\|\left(\vec{y}_{i}-\vec{x}\right) \otimes \vec{d}_{i}\right\|
$$

and we have that

$$
\frac{\partial f_{X}}{\partial x_{j}}=\frac{\left\langle-\vec{e}_{j} \otimes \vec{d}_{i},\left(\vec{y}_{i}-\vec{x}\right) \otimes \vec{d}_{i}\right\rangle}{2\left\|\left(\vec{y}_{i}-\vec{x}\right) \otimes \vec{d}_{i}\right\|}=\frac{\left\langle-\vec{e}_{j} \otimes \vec{d}_{i}, \vec{n}_{i}\right\rangle}{2}=\frac{\left\langle\vec{n}_{i} \otimes \vec{d}_{i}, \vec{e}_{j}\right\rangle}{2} \text { for } j=1,2,3,
$$


where

$$
\vec{n}_{i}=\frac{\left(\vec{y}_{i}-\vec{x}\right) \otimes \vec{d}_{i}}{\left\|\left(\vec{y}_{i}-\vec{x}\right) \otimes \vec{d}_{i}\right\|}
$$

is the unit normal to the triangle in $\mathbb{R}^{3}$. The vector $\vec{n}_{i} \otimes \vec{d}_{i}$ has the same length as $\vec{d}_{i}$ ( $\vec{n}_{i}$ is orthogonal to $\left.\vec{d}_{i}\right)$ and points from the edge belonging to $\vec{d}_{i}$ inside the triangle. Thus we get

$$
\nabla\left(f_{X}\right)=\frac{1}{2} \vec{n}_{i} \otimes \vec{d}_{i}
$$

Summing up over all triangles we get

$$
\nabla(\mathcal{A}(X))=\frac{1}{2} \sum_{i=1}^{k} \vec{n}_{i} \otimes \vec{d}_{i} .
$$

We conclude that the extremality condition for the minimizing cone is given by:

$$
\nabla(\mathcal{A}(X)) \text { points in direction of } \vec{e}_{3} .
$$

Thus the following three statements are equivalent:

1. The projection of $\nabla \mathcal{A}(X)$ on the $\left(x_{1}, x_{2}\right)$-plane vanishes.

2. $\nabla(\mathcal{A}(X)) \otimes \overrightarrow{e_{3}}=\overrightarrow{0}$.

3. $\frac{1}{2} \sum_{i=1}^{k}\left\langle\vec{n}_{i} \otimes \vec{d}_{i}, \vec{e}_{j}\right\rangle=0$ for $j=1,2$.

Since $\mathcal{K}$ is a planar domain in the $\left(x_{1}, x_{2}\right)$-plane we see that $\left(\vec{n}_{i} \otimes \vec{d}_{i}\right) /\left\|\vec{d}_{i}\right\|$ represents the slope of the facet and the direction of the lines of constant slope. Let us now formulate and prove an observation that has been, at least partially, mentioned by A. Pleijel himself.

Theorem 1 Let $\mathcal{K}$ be a closed polygon in the $\left(x_{1}, x_{2}\right)$-plane, which is circumscribing a circle. Then $x^{*}(h)$ is located in the center of the circumscribed circle for every $h>0$.

Remark 3 In Theorem 1 the set $\mathcal{K}$ does not have to be convex (see Figure 3 below).

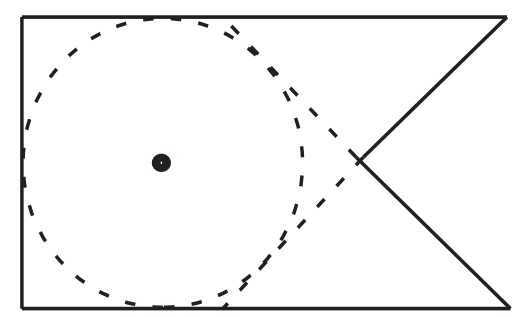

Figure 3: The optimal point in a nonconvex polygon circumscribed to a circle.

\section{Proof of Theorem 1:}

The tangency condition for each side of the polygon implies that all triangles of that cone over $\mathcal{K}$ with apex located over the center of the circle have the same slope. This implies that the projection on the 
$\left(x_{1}, x_{2}\right)$-plane $\Pi_{\left(x_{1}, x_{2}\right)}\left(\vec{n}_{i} \otimes \vec{d}_{i}\right)$ is obtained from $\vec{d}_{i}$ by a $90^{\circ}$-rotation followed by a shrinking of a factor that is independent of $i$. Let us call this linear map $\Phi$. Therefore, we have that

$$
\begin{aligned}
\Pi_{\left(x_{1}, x_{2}\right)}(\nabla(\mathcal{A}(X))) & =\Pi_{\left(x_{1}, x_{2}\right)}\left(\frac{1}{2} \sum_{i=1}^{k} \vec{n}_{i} \otimes \vec{d}_{i}\right)=\frac{1}{2} \sum_{i=1}^{k} \Pi_{\left(x_{1}, x_{2}\right)}\left(\vec{n}_{i} \otimes \vec{d}_{i}\right) \\
& =\frac{1}{2} \sum_{i=1}^{k} \Phi\left(\vec{d}_{i}\right)=\frac{1}{2} \Phi\left(\sum_{i=1}^{k} \vec{d}_{i}\right)=\frac{1}{2} \Phi(\overrightarrow{0})=\overrightarrow{0}
\end{aligned}
$$

since $\mathcal{K}$ is a closed polygon. Obviously the above solution is independent of $h$.

Remark 4 From the assumptions of Theorem 1 we conclude that the solution with minimal surface area coincides with the constant slope solution. The latter one is preferred by architects and engineers for building roofs. To make the latter solution possible one changes from conical surfaces to surfaces having ridges in the common sense; let us observe that, for a generic convex polygon, the bounded slope solution is not a cone.

Remark 5 Theorem 1 also covers the case of triangles. Then the optimal point $x^{*}(h)=\left(x_{1}^{*}, x_{2}^{*}\right)$ is given by the common intersection of the angle bisectors, which also is the center of the inscribed circle.

Remark 6 A simple consequence of Theorem 1 is the fact that for a reflection symmetric (convex) quadrangle the optimal point $x^{*}(h)$ coincides for every $h$ with the center of the inscribed circle.

Remark 7 The assumptions of Theorem 1 imply that the CMIC-set reduces to a singleton in this case.

However, using slightly modified arguments one can show the following result.

Theorem 2 For reflection symmetric hexagons, with two edges parallel to the axis of symmetry, the optimal point $x^{*}(h)$ is independent of the height $h$. This point lies in the intersection of the angle bisectors of the quadrangle constructed by prolonging the two edges passing through the vertices on the axis of symmetry.

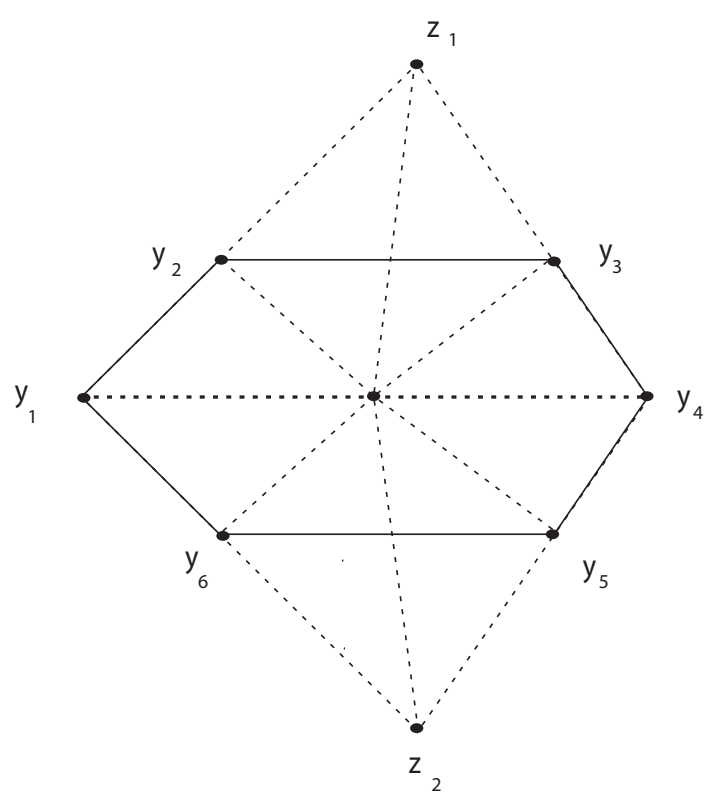

Figure 4: The polygons $\mathcal{K}$ and $\tilde{\mathcal{K}}$. 
A given hexagon $\mathcal{K}=\left\{y_{1}, \ldots, y_{6}\right\}$ can be extended to a reflection symmetric quadrangle $\tilde{\mathcal{K}}=\left\{y_{1}, z_{1}, y_{4}, z_{2}\right\}$. In $\nabla(\mathcal{A}(X))=\frac{1}{2} \sum_{i=1}^{6} \vec{n}_{i} \otimes \vec{d}_{i}$ the horizontal parts of $\vec{n}_{2} \otimes \vec{d}_{2}$ and $\vec{n}_{5} \otimes \vec{d}_{5}$ cancel each other for any choice of the apex on the line of symmetry. By similarity there is a common factor $\lambda$ such that $\vec{d}_{1}=\lambda \overrightarrow{\tilde{d}}_{1}, \vec{d}_{3}=\lambda \overrightarrow{\tilde{d}}_{2}$, $\vec{d}_{4}=\lambda \overrightarrow{\tilde{d}}_{3}$ and $\vec{d}_{6}=\lambda \overrightarrow{\tilde{d}}_{4}$. Obviously $\vec{n}_{1}=\overrightarrow{\tilde{n}}_{1}, \vec{n}_{3}=\overrightarrow{\tilde{n}}_{2}, \vec{n}_{4}=\overrightarrow{\tilde{n}}_{3}$ and $\vec{n}_{6}=\overrightarrow{\tilde{n}}_{4}$. Choosing $x^{*}(h)$ as the common intersection of the angle bisectors of the polygon $\tilde{\mathcal{K}}$ as above we get

$$
\begin{aligned}
\overrightarrow{0} & =\Pi_{\left(x_{1}, x_{2}\right)}\left(\frac{1}{2} \sum_{i=1}^{4} \overrightarrow{\tilde{n}}_{i} \otimes \overrightarrow{\tilde{d}}_{i}\right) \\
& =\Pi_{\left(x_{1}, x_{2}\right)} \frac{1}{2 \lambda}\left(\vec{n}_{1} \otimes \vec{d}_{1}+\vec{n}_{3} \otimes \vec{d}_{3}+\vec{n}_{4} \otimes \vec{d}_{4}+\vec{n}_{6} \otimes \vec{d}_{6}\right) .
\end{aligned}
$$

However, remembering the cancellation condition for $\vec{n}_{2} \otimes \vec{d}_{2}$ and $\vec{n}_{5} \otimes \vec{d}_{5}$ we see that the chosen location of $x^{*}(h)$ is also the optimal one for $\mathcal{K}$. As in Theorem 1 , the choice of $x^{*}(h)$ is independent of the height $h$. However, in contrast to the previous result, the solution here is not - in general - a constant slope solution: the slope of facets over $\vec{d}_{2}$ and $\vec{d}_{5}$ may be different from the slope of the other four facets.

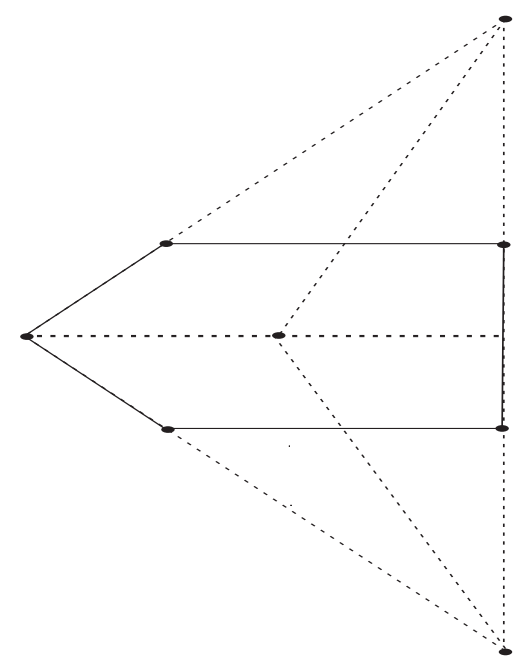

Figure 5: How to deal with a pentagon.

In the same way one can proceed in the case of a reflection symmetric pentagon having two edges parallel to the axis of symmetry. In this case one edge must be orthogonal to the axis of symmetry. As before, we can extend the pentagon in a triangle by prolonging the two edges passing through the vertex on the axis of symmetry and the edge perpendicular to this axis. The intersection of the angle bisectors of the triangle gives us the optimal point $x^{*}(h)$ for every $\left.h \in\right] 0, \infty[$. This can be shown in the same way as in the case of the hexagon. Thus we have:

Theorem 3 For a reflection symmetric pentagon having two edges parallel to the axis of symmetry the optimal point $x^{*}(h)$ is independent of $h$. This point lies in the intersection of the angle bisectors of the triangle constructed by prolonging the two edges passing through the vertex on the axis of symmetry and the edge perpendicular to this axis.

Let us now turn to reflection symmetric trapezoids. Up to similarities the following data describe the situation in full generality (compare Figure 6). 
As a preliminary result we have:

$$
\prod_{\left(x_{1}, x_{2}\right)}\left(\vec{n}_{1} \otimes \vec{d}_{1}+\vec{n}_{3} \otimes \vec{d}_{3}\right)=\frac{2(\lambda-1) \sin (\alpha) \tan (\alpha)}{\sqrt{\sin ^{2}(\alpha)+\tan ^{2}(\beta)}}(1,0)^{T} .
$$

For the given situation this term does not depend on the location of the apex on the line through the origin in direction of $\vec{x}$, but clearly $h$ varies with that location. Setting $\vec{w}_{2}:=\vec{x}-(\lambda, 0,0)$ we get

$$
\begin{aligned}
\vec{n}_{2} \otimes \vec{d}_{2} & =\left\|\overrightarrow{d_{2}}\right\| \frac{\vec{w}_{2}}{\left\|\vec{w}_{2}\right\|} \\
& =\frac{2 \lambda \tan (\alpha)}{\sqrt{\left(\lambda-x_{1}\right)^{2}+x_{1}^{2} \tan ^{2}(\beta)}}\left(-\left(\lambda-x_{1}\right), 0, x_{1} \tan (\beta)\right)^{T}
\end{aligned}
$$

and similarly

$$
\vec{n}_{4} \otimes \vec{d}_{4}=\frac{2 \tan (\alpha)}{\sqrt{\left(x_{1}-1\right)^{2}+x_{1}^{2} \tan ^{2}(\beta)}}\left(\left(x_{1}-1\right), 0, x_{1} \tan (\beta)\right)^{T}
$$

Hence

$$
\Pi_{\left(x_{1}, x_{2}\right)}\left(\vec{n}_{2} \otimes \vec{d}_{2}+\vec{n}_{4} \otimes \vec{d}_{4}\right)=2 \tan (\alpha)\left(\frac{1}{\sqrt{1+\left(\frac{x_{1}}{x_{1}-1}\right)^{2} \tan ^{2}(\beta)}}-\frac{\lambda}{\sqrt{1+\left(\frac{x_{1}}{\lambda-x_{1}}\right)^{2} \tan ^{2}(\beta)}}\right)(1,0)^{T} .
$$

Therefore, the minimality condition reads:

$$
0=\frac{2(\lambda-1) \sin (\alpha) \tan (\alpha)}{\sqrt{\sin ^{2}(\alpha)+\tan ^{2}(\beta)}}+2 \tan (\alpha)\left(\frac{1}{\sqrt{1+\left(\frac{x_{1}}{x_{1}-1}\right)^{2} \tan ^{2}(\beta)}}-\frac{\lambda}{\sqrt{1+\left(\frac{x_{1}}{\lambda-x_{1}}\right)^{2} \tan ^{2}(\beta)}}\right)
$$

which can be simplified to

$$
0=\frac{(\lambda-1)}{\sqrt{1+\frac{\tan ^{2}(\beta)}{\sin (\alpha)}}}+\left(\frac{1}{\sqrt{1+\left(\frac{x_{1}}{x_{1}-1}\right)^{2} \tan ^{2}(\beta)}}-\frac{\lambda}{\sqrt{1+\left(\frac{x_{1}}{\lambda-x_{1}}\right)^{2} \tan ^{2}(\beta)}}\right) .
$$

Substituting $h=x_{1} \tan (\beta)$ we get:

$$
0=\frac{(\lambda-1)}{\sqrt{1+\frac{h^{2}}{x_{1} \sin (\alpha)}}}+\left(\frac{1}{\sqrt{1+\left(\frac{h}{x_{1}-1}\right)^{2}}}-\frac{\lambda}{\sqrt{1+\left(\frac{h}{\lambda-x_{1}}\right)^{2}}}\right)
$$

It is now possible to characterize when the point $\left(x_{1}^{*}, x_{2}^{*}\right)=\left(x_{1}^{*}, 0\right)$ is located at the midpoint between $(1,0)$ and $(\lambda, 0)$, and to come to a familiar situation. The solution of $(7)$ gives $x_{1}=\frac{\lambda+1}{2}$, and then

$$
0=\frac{(\lambda-1) \sin (\alpha)}{\sqrt{\sin ^{2}(\alpha)+\tan ^{2}(\beta)}}-\frac{\lambda-1}{\sqrt{1+\left(\frac{\lambda+1}{\lambda-1}\right)^{2} \tan ^{2}(\beta)}} .
$$


Then we have

$$
\sin ^{2}(\alpha)\left(\frac{\lambda+1}{\lambda-1}\right)^{2}=1
$$

For $\lambda=(1+\sin (\alpha)) /(1-\sin (\alpha))$ the quadrangle is circumscribed to a circle. (In this situation we have $x_{1}+r=\lambda, x_{1}-r=1$ with $r$ being the radius and $r=x_{1} \sin (\alpha)$.)

We can summarize as follows.

Theorem 4 For the reflection symmetric trapezoids introduced before, $\left(x_{1}^{*}, 0\right)=((\lambda+1) / 2,0)$ for every $h$ if and only if $\lambda=(1+\sin (\alpha)) /(1-\sin (\alpha))$ and the quadrangle is circumscribed to a circle.

Are there other cases of reflection symmetric trapezoids where $\left(x_{1}^{*}, x_{2}^{*}\right)=\left(x_{1}^{*}, 0\right)$ does not move with $h$ ? If such a situation occurs, then the point does not move with $h^{2}$ either. Hence considering differentiable (twice) dependence of $X^{*}$ from $h^{2}$ and assuming the first two derivatives w.r.t. $h^{2}$ of $\mathcal{A}$ equal to 0 we get from (7):

$$
\begin{aligned}
0 & =\left\langle(\lambda-1,1,-\lambda)^{T},\left(c_{1}, c_{2}, c_{3}\right)^{T}\right\rangle \\
& =\left\langle\left(\frac{\lambda-1}{x_{1}^{2} \sin ^{2}(\alpha)+h^{2}}, \frac{1}{\left(x_{1}-1\right)^{2}+h^{2}}, \frac{-\lambda}{\left(\lambda-x_{1}\right)^{2}+h^{2}}\right)^{T},\left(c_{1}, c_{2}, c_{3}\right)^{T}\right\rangle \\
& =\left\langle\left(\frac{\lambda-1}{\left(x_{1}^{2} \sin ^{2}(\alpha)+h^{2}\right)^{2}}, \frac{1}{\left(\left(x_{1}-1\right)^{2}+h^{2}\right)^{2}}, \frac{-\lambda}{\left(\left(\lambda-x_{1}\right)^{2}+h^{2}\right)^{2}}\right)^{T},\left(c_{1}, c_{2}, c_{3}\right)^{T}\right\rangle
\end{aligned}
$$

with

$$
c_{1}=\frac{1}{\sqrt{1+\frac{h^{2}}{x_{1}^{2} \sin ^{2}(\alpha)}}}, c_{2}=\frac{1}{\sqrt{1+\frac{h^{2}}{\left(x_{1}-1\right)^{2}}}}, c_{3}=\frac{1}{\sqrt{1+\frac{h^{2}}{\left(\lambda-x_{1}\right)^{2}}}} .
$$

The vector on the right hand side of the scalar products above does not vanish, hence the left hand side must be linearly dependent. Extracting from the corresponding vanishing determinant the nonzero factors $\lambda-1$ and $-\lambda$ we get

$$
\begin{gathered}
0=\operatorname{det}\left(\begin{array}{ccc}
\frac{1}{1} & 1 & 1 \\
\frac{1}{\left(x_{1}^{2} \sin ^{2}(\alpha)+h^{2}\right)} & \frac{1}{\left(\left(x_{1}-1\right)^{2}+h^{2}\right)} & \frac{1}{\left(\left(\lambda-x_{1}\right)^{2}+h^{2}\right)} \\
\frac{1}{\left(x_{1}^{2} \sin ^{2}(\alpha)+h^{2}\right)^{2}} & \frac{1}{\left(\left(x_{1}-1\right)^{2}+h^{2}\right)^{2}} & \frac{1}{\left(\left(\lambda-x_{1}\right)^{2}+h^{2}\right)^{2}}
\end{array}\right) \\
=\left(\frac{1}{\left(x_{1}^{2} \sin ^{2}(\alpha)+h^{2}\right)}-\frac{1}{\left(\left(x_{1}-1\right)^{2}+h^{2}\right)}\right)\left(\frac{1}{\left(\left(x_{1}-1\right)^{2}+h^{2}\right)}-\frac{1}{\left(\left(\lambda-x_{1}\right)^{2}+h^{2}\right)}\right) \\
\times\left(\frac{1}{\left(\left(\lambda-x_{1}\right)^{2}+h^{2}\right)}-\frac{1}{\left(x_{1}^{2} \sin ^{2}(\alpha)+h^{2}\right)}\right) .
\end{gathered}
$$

Thus we get the following alternatives:

$$
x_{1} \sin (\alpha)=x_{1}-1 \text { or } x_{1}-1=\lambda-x_{1} \text { or } \lambda-x_{1}=x_{1} \sin (\alpha) \text {. }
$$

Summarizing the observations above we see that:

- When all identities in (8) are violated, then the critical point changes with $h$.

- If at least one of the identities (8) is satisfied, together with (7), the point $X^{*}$ is stable up to the second order with respect to $h$. 


\section{Position of the optimal point for general reflection symmetric convex polygons}

As we have seen in the case of the trapezoid the location of $x^{*}(h)$ might differ as $h$ varies. It is the purpose of this section to investigate the location of $x^{*}(h)$ as $h \rightarrow \infty$ and as $h \rightarrow 0$. We shall show the following Teorem.

Theorem 5 Assume that $\mathcal{K}$ is a reflection symmetric polygon with only one axis of symmetry and $k$ vertices. If $x_{\infty}^{*}=\lim _{h \rightarrow \infty} x^{*}(h)$, then $x_{\infty}^{*}$ is explicitly given by formula (10) below. If $x_{0}^{*}=\lim _{h \rightarrow 0} x^{*}(h)$, then $x_{0}^{*}=\left(x_{1}^{*}(0), 0\right)$ is in the interior of $\mathcal{K}$ and $x_{1}^{*}(0)$ satisfies the necessary condition (11) given below.

To understand the proof of the Theorem, let us first look at two concrete examples and let us calculate the optimal points numerically for some very large and some very small heights.

Example 1 Let us consider the polygon with vertices $(-2,0),(-2,1),(-1.6,2),(7,2),(7.5,0),(7,-2)$, $(-1.6,-2)$ and $(-2,-1)$.

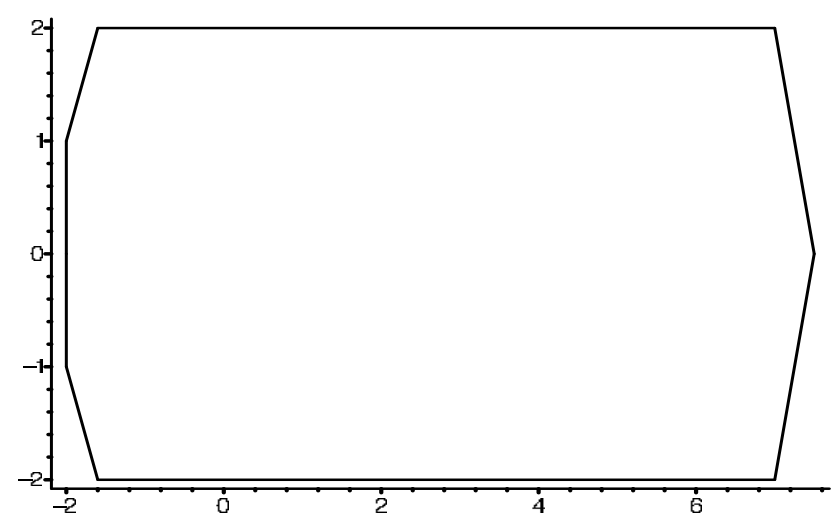

Figure 7: Where is the optimal point for this hexagon?

Using an implementation by Greg Spradlin [5] of the Nelder-Mead Method [4] with initial simplex y1 := $(1.5,0), y 2:=(2,0), y 3:=(2,0.5)$ for Maple 9.5, we get the following optimal points for different values of $h$.

\begin{tabular}{|c|c|c|}
\hline Height $h$ & Coordinates of the optimal point $x^{*}(h) \in \mathbb{R}^{2}$ & stopping tolerance \\
\hline 100 & $(2.673004985, .1869916924 \mathrm{e}-2)$ & $1.0 \cdot 10^{-5}$ \\
\hline 10 & $(2.668712891, .3709755827 \mathrm{e}-3)$ & $1.0 \cdot 10^{-5}$ \\
\hline 1 & $(2.668638495,-.2638438224 \mathrm{e}-4)$ & $1.0 \cdot 10^{-7}$ \\
\hline
\end{tabular}

Here we have not made use of the fact that the domain is symmetric. On the contrary, we used the NelderMead method to minimize a function in $\mathbb{R}^{2}$ directly.

Example 2 Let us now turn to a trapezoid with vertices $(-2,-1),(-2,1),(7,2)$ and $(7,-2)$ given in Figure 8. 


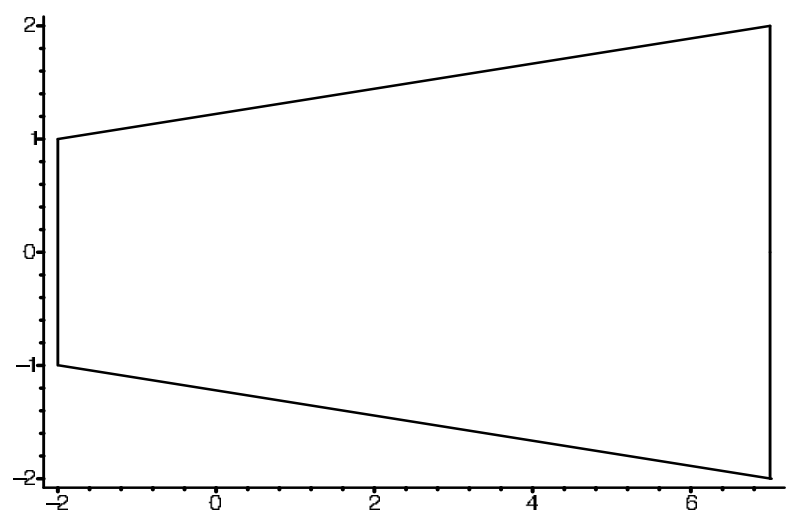

Figure 8: Where is the optimal point for this trapezoid?

Here the numerical computations with the stopping tolerance $1.0 \cdot 10^{-7}$ and the same initial simplex as above give us:

\begin{tabular}{|c|c|}
\hline Height $h$ & Coordinates of the optimal point $x^{*}(h) \in \mathbb{R}^{2}$ \\
\hline 100 & $(3.467648968, .6963578119 \mathrm{e}-3)$ \\
\hline 10 & $(3.584020263, .1278804851 \mathrm{e}-3)$ \\
\hline 1 & $(4.542435792,-.2093678430 \mathrm{e}-4)$ \\
\hline 0.1 & $(4.638908112,-.7018900381 \mathrm{e}-3)$ \\
\hline
\end{tabular}

In this case the numerical results suggest, too, that the optimal points converges, for $h \rightarrow 0$ and for $h \rightarrow \infty$ to two distinct points in the interior of the domain $\mathcal{K}$.

Now we concentrate on the case of a reflection symmetric polygon $\mathcal{K}=\left\{y_{1}, \ldots, y_{k}\right\}(k \geq 8)$. Without loss of generality we assume that $k$ is even and that the polygon is reflection symmetric to the $x_{2}$-axis. Furthermore, we assume that there are two edges that are parallel to the symmetry axis and that $y_{1}$ and $y_{\frac{k}{2}+1}$ lie on this axis. The extremality condition reduces to the equation

$$
\frac{1}{2} \sum_{i=1}^{k}\left\langle\vec{n}_{i} \otimes \vec{d}_{i}, \vec{e}_{1}\right\rangle=0
$$

Therefore, critical points must satisfy

$$
\sum_{i=1}^{k}\left(\prod_{\substack{j \neq i \\ j=1}}^{k}\left\|\left(\vec{y}_{j}-\vec{x}\right) \otimes \vec{d}_{j}\right\|\right)\left\langle\left(\left(\vec{y}_{i}-\vec{x}\right) \otimes \vec{d}_{i}\right) \otimes \vec{d}_{i}, \vec{e}_{1}\right\rangle=0 .
$$

The symmetry assumption allows us to conclude that the optimal apex satisfies

$$
\sum_{i=1}^{\frac{k}{2}}\left(\prod_{\substack{j \neq i \\ j=1}}^{\frac{k}{2}}\left\|\left(\vec{y}_{j}-\vec{x}\right) \otimes \vec{d}_{j}\right\|\right)\left\langle\left(\left(\vec{y}_{i}-\vec{x}\right) \otimes \vec{d}_{i}\right) \otimes \vec{d}_{i}, \vec{e}_{1}\right\rangle=0 .
$$

However, since two edges are parallel to the symmetry axis, there exists $i^{\star} \in\left\{2, \ldots, \frac{k}{2}-1\right\}$ such that

$$
\left\langle\left(\left(\vec{y}_{i^{\star}}-\vec{x}\right) \otimes \vec{d}_{i^{\star}}\right) \otimes \vec{d}_{i^{\star}}, \vec{e}_{1}\right\rangle=0
$$


so there are only $\frac{k}{2}-1$ addends in this case. To simplify notations we set:

$$
\begin{gathered}
\sum_{\substack{i \neq i^{*} \\
i=1}}^{\frac{k}{2}}\left(\prod_{\substack{j \neq i, j \neq i^{*} \\
j=1}}^{\frac{k}{2}} \sqrt{\left(a_{j} x_{1}+b_{j}\right)^{2}+c_{j} h^{2}}\right) a_{i}\left(a_{i} x_{1}+b_{i}\right) \\
:= \\
\sum_{\substack{i \neq i^{*} \\
i=1}}^{\frac{k}{2}}\left(\prod_{\substack{j \neq i, j \neq i^{*} \\
j=1}}^{\frac{k}{2}}\left\|\left(\vec{y}_{j}-\vec{x}\right) \otimes \vec{d}_{j}\right\|\right)\left\langle\left(\left(\vec{y}_{i}-\vec{x}\right) \otimes \vec{d}_{i}\right) \otimes \vec{d}_{i}, \vec{e}_{1}\right\rangle
\end{gathered}
$$

where $a_{i}:=\left(y_{2, i}-y_{2, i+1}\right), b_{i}:=-\left(y_{2, i} y_{1, i+1}-y_{1, i} y_{2, i+1}\right)$ and $c_{i}:=\left(y_{1, i}-y_{1, i+1}\right)^{2}+\left(y_{2, i}-y_{2, i+1}\right)^{2}$. To simplify notation even further let us set $A_{j}\left(x_{1}\right)=a_{j} x_{1}+b_{j}$ for all $j \in\left\{1, \ldots \frac{k}{2}\right\}$. Now we define the function

$$
\mathcal{Q}(x, h):=\sum_{i=1}^{\frac{k}{2}}\left(\prod_{\substack{j \neq i, j \neq i^{*} \\ j=1}}^{\frac{k}{2}} \sqrt{A_{j}^{2}(x)+c_{j} h^{2}}\right) a_{i} A_{i}(x) .
$$

\subsection{The limit $h \rightarrow \infty$}

The function $\mathcal{Q}(x, h)$ is continuous in $h$. Of course it is now possible to consider the limit $h \rightarrow \infty$. In this case one gets

$$
\begin{aligned}
\lim _{h \rightarrow \infty} \mathcal{Q}\left(x_{1}, h\right) & =\lim _{h \rightarrow \infty} \sum_{\substack{i \neq i^{*} \\
i=1}}^{\frac{k}{2}}\left[\left(\prod_{\substack{j \neq i, j \neq i^{*} \\
j=1}}^{\frac{k}{2}} \sqrt{A_{j}^{2}\left(x_{1}\right)+c_{j} h^{2}}\right) a_{i} A_{i}\left(x_{1}\right)\right] \\
& \sim \lim _{h \rightarrow \infty} \sum_{\substack{i \neq i^{*} \\
i=1}}^{\frac{k}{2}}\left[\left(\prod_{\substack{j \neq i \\
j=1}}^{\frac{k}{2}} \sqrt{c_{j}}\right) h^{\frac{k-2}{2}} a_{i} A_{i}\left(x_{1}\right)\right]+O(1) .
\end{aligned}
$$

as $h \rightarrow \infty$. Remembering that $A_{i}\left(x_{1}\right)=a_{i} x_{1}+b_{i}$ we see that this time the equation

$$
\sum_{\substack{i \neq i^{*} \\ i=1}}^{\frac{k}{2}}\left[\left(\prod_{\substack{j \neq i, j \neq i^{*} \\ j=1}}^{\frac{k}{2}} \sqrt{c_{j}}\right) a_{i} A_{i}\left(x_{1}\right)\right]=0
$$

is affine in $x_{1}$ and gives us the algebraic coordinates of the optimal apex. This is explicitly given by

$$
\left(-\frac{\sum_{\substack{i \neq i^{*} \\ i=1}}^{\frac{k}{2}}\left[\left(\prod_{\substack{j \neq i, j \neq i^{*} \\ j=1}}^{\frac{k}{2}} \sqrt{c_{j}}\right) a_{i} b_{i}\right]}{\sum_{\substack{i \neq i^{*} \\ i=1}}^{\frac{k}{2}}\left[\left(\prod_{\substack{j \neq i, j \neq i^{*} \\ j=1}}^{\frac{k}{2}} \sqrt{c_{j}}\right) a_{i}^{2}\right]}, 0\right) .
$$

Calculating this value for Example 1 we get in the limit $h \rightarrow \infty$ the optimal point

$$
\left(-1-\frac{\sqrt{29} \sqrt{17}}{\sqrt{29} \sqrt{17}+12 \sqrt{17})-60 \sqrt{29}}, 0\right) .
$$


Doing the same calculations also for Example 2 we see that in the case of the given Trapezoid the optimal point in the limit $h \rightarrow \infty$ is given by

$$
\left(-1+\frac{2 \sqrt{82}}{(26 \sqrt{82}-22)}, 0\right)
$$

\subsection{The limit $h \rightarrow 0$}

To analyze the case $h \rightarrow 0$ we use the Taylor expansion of square root and see that for all $h$

$$
\begin{aligned}
\mathcal{Q}\left(x_{1}, h\right) & \approx \sum_{\substack{i \neq i^{*} \\
i=1}}^{\frac{k}{2}}\left[\left(\prod_{\substack{j \neq i, j \neq i^{*} \\
j=1}}^{\frac{k}{2}}\left(\left|A_{j}\left(x_{1}\right)\right|+\frac{c_{j}\left|A_{j}\left(x_{1}\right)\right|}{2 A_{j}^{2}\left(x_{1}\right)} h^{2}+O\left(h^{4}\right)\right)\right] a_{i} A_{i}\left(x_{1}\right)\right] \\
& \approx\left[\sum_{\substack{i \neq i^{*} \\
i=1}}^{\frac{k}{2}}\left(a_{i} \operatorname{sign}\left(A_{i}\left(x_{1}\right)\right) \cdot \sum_{\substack{l \neq i, k \neq i^{*} \\
l \neq 1}}^{k / 2} \frac{c_{l}}{A_{l}^{2}\left(x_{1}\right)}\right) \prod_{\substack{j \neq i^{*} \\
j=1}}^{\frac{k}{2}}\left|A_{j}\left(x_{1}\right)\right|\right] \frac{h^{2}}{2}+O\left(h^{4}\right) \\
& =\left\{-\left[\sum_{\substack{i \neq i^{*} \\
i=1}}^{\frac{k}{2}} \frac{a_{i} c_{i} \operatorname{sign}\left(A_{i}\left(x_{1}\right)\right)}{A_{i}^{2}\left(x_{1}\right)}\right] \prod_{\substack{j \neq i^{*} \\
j=1}}^{\frac{k}{2}}\left|A_{j}\left(x_{1}\right)\right|\right\} \frac{h^{2}}{2}+O\left(h^{4}\right) .
\end{aligned}
$$

Here we used the fact that

$$
\sum_{\substack{i \neq i^{*} \\ i=1}}^{\frac{k}{2}} a_{i} \operatorname{sign}\left(A_{i}\left(x_{1}\right)\right)=0
$$

twice. Therefore, for small $h$ the $x_{1}$-coordinate of the optimal point converges either to a root of

$$
\sum_{\substack{i \neq i^{*} \\ i=1}}^{\frac{k}{2}} \frac{a_{i} c_{i} \operatorname{sign}\left(A_{i}\left(x_{1}\right)\right)}{A_{i}^{2}\left(x_{1}\right)}=0
$$

or to a root of

$$
\prod_{\substack{j \neq i^{*} \\ j=1}}^{\frac{k}{2}}\left|A_{j}\left(x_{1}\right)\right|
$$

The roots of the last product are given by $z_{j}=-b_{j} / a_{j}, j=\left\{1, \ldots, \frac{k}{2}\right\} \backslash\left\{i^{*}\right\}$. Therefore, if the optimal point coincides with one of these points $\left(z_{j}, 0\right)$ as $h \rightarrow 0$, it must coincide with either $y_{1}=\left(-b_{1} / a_{1}, 0\right)$ or $y_{\frac{k}{2}+1}=\left(-b_{\frac{k}{2}} / a_{\frac{k}{2}}, 0\right)$, since all the other candidates lie outside of $\mathcal{K}$.

Remark 8 For all $j=\left\{1, \ldots, \frac{k}{2}\right\} \backslash\left\{i^{*}\right\}$ we have that the points $\left(y_{1, i}, y_{2, i}\right)$ and $\left(y_{1, i+1}, y_{2, i+1}\right)$ lie on a line that intersects the symmetry axis in the points $z_{j}$. For $k / 2-1$ odd, the convexity of $\mathcal{K}$ implies that all roots lie outside of the interval $\left[y_{1,1}, y_{1, \frac{k}{2}+1}\right]$. Thus the only candidates for the location of the optimal point are $y_{1}$ and $y_{\frac{k}{2}+1}$, since all other possible candidates do not belong to $\overline{\mathcal{K}}$.

However, we see that

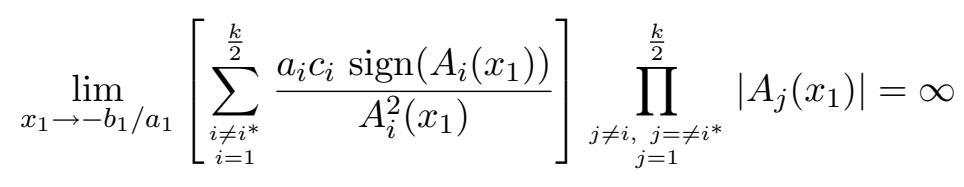




$$
\lim _{x_{1} \rightarrow-b_{\frac{k}{2}} / a_{\frac{k}{2}}}\left[\sum_{\substack{i \neq i^{*} \\ i=1}}^{\frac{k}{2}} \frac{a_{i} c_{i} \operatorname{sign}\left(A_{i}\left(x_{1}\right)\right)}{A_{i}^{2}\left(x_{1}\right)}\right] \prod_{\substack{j \neq i \\ j=1}}^{\frac{k}{2}}\left|A_{j}\left(x_{1}\right)\right|=\infty .
$$

Thus, neither one can be the optimal point in the limit $h \rightarrow 0$. Therefore, we conclude that in the limit $h \rightarrow 0$ the $x_{1}$-coordinate of the optimal point will solve the equation

$$
\sum_{\substack{i \neq i^{*} \\ i=1}}^{\substack{2}} \frac{a_{i} c_{i} \operatorname{sign}\left(A_{i}\left(x_{1}\right)\right)}{A_{i}^{2}\left(x_{1}\right)}=0
$$

In Example 1 equation (11) turns into

$$
\frac{8}{\left(2 x_{1}+4\right)^{2}}+\frac{232}{25\left(2 x_{1}+\frac{24}{5}\right)^{2}}-\frac{68}{\left(-4 x_{1}+30\right)^{2}}=0 .
$$

Using MAPLE once again we see that this fourth order equation in $x_{1}$ has only one zero in the interval $(-2,7.5)$ namely $x_{1}=2.668558840$. This is consistent with the values in the table of Example 1. Looking again at Example 2 we see that (11) turns into

$$
\frac{164}{\left(x_{1}+11\right)^{2}}-\frac{64}{\left(-4 x_{1}+28\right)^{2}}+\frac{8}{\left(2 x_{1}+4\right)^{2}}=0
$$

in this case and calculating the root of this forth order equation in $x_{1}$ gives us $x_{1}=4.636652188$ as the only zero in the interval $(-2,7)$. This is again consistent with the value in the table of Example 2 .

\subsection{Remarks on general convex domains}

We still have some unanswered questions. What happens if we drop the symmetry assumption? We expect that the following is true:

Conjecture 1 Let $\mathcal{K}$ be an arbitrary convex domain. Further suppose that the CMIC-set is not a singleton. For a given height $h$ the optimal point $x^{*}(h)$ will always lie on the $C M I C$-set.

On the other hand, we have no suggestion what happens when the CMIC-set is a singleton like in the case of the Trapezoid. The characterization of the limiting points by B. Cheng [1] is the only result available in this situation so far. Thus it would be interesting to know the answer to Pleijel's question also in this cases. But let us close this article with the following:

Conjecture 2 A sufficient condition for convex domains $\mathcal{K}$ to have an optimal point, that does not depend on a given height, is that its support function solves the necessary condition (3).

Acknowledgement: The authors thank Bernd Wegner (Berlin) and the referee for their helpful comments and suggestions.

\section{References}

[1] B. N. Cheng (1992) On a problem of A. Pleijel. Geometriae dedicata 44: 139 - 145.

[2] H. T. Croft, K. J. Falconer and R. K. Guy (1991) Unsolved problems in geometry. Berlin: Springer. 
[3] A. Pleijel (1955) Problem 16. Math. Scand. 3: 306.

[4] A. Quarteroni, R. Sacco and E. Saleri (2002) Numerische Mathematik 1. Springer-Verlag, Berlin, Heidelberg, New York.

[5] G. Spradlin: http://www.mapleapps.com/categories/mathematics/Operations\%20Research /html/neldermead.html 